OPEN ACCESS

Edited by:

Gary Sinoff

University of Haifa, Israel

Reviewed by:

Miguel Germán Borda,

Stavanger University Hospital, Norway Juan José Solano-Jaurrieta,

Servicio de Salud del Principado de

Asturias (SESPA), Spain

*Correspondence:

Miao Liu

liumiaolmbxb@163.com

Yao He

yhe301@x263.net

tThese authors have contributed equally to this work

Specialty section:

This article was submitted to

Geriatric Medicine,

a section of the journal

Frontiers in Medicine

Received: 22 September 2020

Accepted: 26 January 2021

Published: 16 February 2021

Citation:

Wang S, Jia W, Yang S, Han K, Cao W, Ren X, Li J, Tai P, Kou F, Liu M and $\mathrm{He} Y$ (2021) The Role of BMl and

Blood Pressure in the Relationship

Between Total Cholesterol and Disability in Chinese Centenarians: A

Cross-Sectional Study.

Front. Med. 8:608941.

doi: 10.3389/fmed.2021.608941

\title{
The Role of BMI and Blood Pressure in the Relationship Between Total Cholesterol and Disability in Chinese Centenarians: A Cross-Sectional Study
}

\begin{abstract}
Shengshu Wang ${ }^{1 \dagger}$, Wangping Jia ${ }^{1 \dagger}$, Shanshan Yang ${ }^{2 \dagger}, \mathrm{Ke} \mathrm{Han}^{1}$, Wenzhe Cao ${ }^{1}$, Xueling Ren ${ }^{3}$, Jing $\mathrm{Li}^{4}$, Penggang Tai ${ }^{5}$, Fuyin $\mathrm{Kou}^{5}$, Miao Liu ${ }^{6 *}$ and $\mathrm{Yao} \mathrm{He}^{1,7 *}$
\end{abstract}

${ }^{1}$ Institute of Geriatrics, Beijing Key Laboratory of Aging and Geriatrics, National Clinical Research Center for Geriatrics Diseases, Second Medical Center of Chinese PLA General Hospital, Beijing, China, ${ }^{2}$ Department of Disease Prevention and Control, The 1st Medical Center, Chinese PLA General Hospital, Beijing, China, ${ }^{3}$ Department of Respiratory, The 2nd Medical Center, Chinese People's Liberation Army General Hospital, Beijing, China, ${ }^{4}$ Medical Service Department, The 5th Medical Center, Chinese People's Liberation Army General Hospital, Beijing, China, ${ }^{5}$ Medical Service Department, Chinese People's Liberation Army General Hospital, Beijing, China, ${ }^{6}$ Department of Statistics and Epidemiology, Graduate School of Chinese PLA General Hospital, Beijing, China, ${ }^{7}$ State Key Laboratory of Kidney Diseases, Chinese People's Liberation Army General Hospital, Beijing, China

Background: Lower serum lipid metabolism might be associated with the decline of activity of daily living in the extreme longevity group. However, studies on models and possible paths of this correlation between total cholesterol (TC) and disability in centenarians are scarce. The aim of this study was to verify this correlation and explore the mediating effect of BMl and blood pressure on this relationship in Hainan centenarians.

Methods: We conducted a cross-sectional analysis of 1002 centenarians from the China Hainan Centenarians Cohort Study (CHCCS). Data on demographics, anthropometry data, lifestyle, and TC levels were collected through interviews, physical examinations, and laboratory tests. The Barthel index and Lawton index, measuring the disability status, were used to estimate the activity of daily living (ADL) and instrumental activity of daily living (IADL). A multivariable logistic regression model was used to explore the correlation between disability and TC levels. Mediation analyses were used to explore the both direct and indirect effects of TC level on disability.

Results: After adjusting for covariates, with $1 \mathrm{mmol} / \mathrm{L}$ increment in TC, the adjusted odds ratios (ORs) of $A D L$ severe disability and $A D L$ moderate \& severe disability were 0.789(95\%Cl: 0.650-0.959) and 0.822(95\%Cl: 0. 0.699-0.966), respectively. There was a significant declining trend in the prevalence of different types of disability with increment in TC. The correlation was more pronounced among Hainan female centenarians. In the analysis of mediating effect among the female population, BMl significantly mediated 
the effect of TC levels on different types of disability. BMI and SBP, as chain mediators, multiply and chain mediated the effect of TC levels on IADL.

Conclusion: Low TC levels might be correlated with a higher frequency of disability in female centenarians, and this correlation might be mediated by BMl and blood pressure.

Keywords: total cholesterol, activities of daily living, disability, correlation, mediating effect

\section{INTRODUCTION}

It is well-established that population aging is one of the significant challenges for many countries, especially for China (1). The sharp increase in older populations poses a major challenge to health and social security systems in that older people have a higher prevalence of disability (2-4). Activities of daily living (ADL) refers to necessary activities that people undertake routinely for meeting the needs of daily life, and it was usually used to estimate the decline or even loss of body function among older adults (5), while instrumental activity of daily living (IADL) is regarded as a significant indicator to assess the independence of living in society (6). Disability is often known as a problem affecting old people aged 65 or older and the effects of these problems usually increase with age. Considering the specificity of extreme longevity, ADL disability in the general old population cannot precisely represent the centenarians.

Hypercholesterolemia, a major causal risk factor for cardiovascular events, is considered as risk factors for longevity (7-9). However, in an older population, there is no consensus concerning the health impact of hypercholesterolemia on longevity and disability. Studies have shown that higher triglyceride may be related to better ADL among the older population $(10,11)$, and even higher lipid profiles are beneficial to longevity and functional performance among the older population (12-15). In addition, the influence pathway and physiological mechanism of the possible effect of blood lipids on disability were complex and uncertain. Moreover, no epidemiological evidence of the correlation and correlation ways of TC levels and disability, such as ADL and IADL, in centenarians.

The aims of the current study are to explore the possible correlation and potential correlated paths of TC level and disability in centenarians. We used data from the China Hainan Centenarians Cohort Study (CHCCS), which includes complete samples of community-based centenarians in Hainan, China.

\section{MATERIALS AND METHODS}

\section{Subjects}

The data of this study were from the cross-sectional survey of CHCCS from 2014-2016. CHCCS is an ongoing whole-samples centenarians survey in Hainan, China, which was reported elsewhere (16). The first survey wave has been investigated from June 2014 to December 2016 via face-to-face investigation. It was carried out in a complete sample study, containing 1,811 living centenarians in 2014 according to the household register provided by the civil affairs bureau. We excluded 338 centenarians for whom we could not be reached based on the contact information provided. We also excluded individuals who were not conscious and could not perform the questionnaire interviews, physical health examination, and blood collection. A rigorous age validation process was conducted to prevent participants from exaggerating their age (16). Of 1,473 eligible individuals, a total of 1,002 centenarians aged 100 to 116 years, living in community, at enrollment in baseline survey were assessed for study eligibility. The study was approved by the Ethics Committee of the Chinese People's Liberation Army General Hospital (approval number: 301hn11201601). All centenarians signed the written informed consent forms.

\section{ADL Assessment}

Barthel Index (5), and Lawton IADL Scale (17) were used to estimate whether it is a disability. Participants had been inquired if they could finish the items from the ADL scales. The Barthel ADL Index, containing 10 items with 0 points for inability to 10 or 15 points for complete independence, were summed to give a score from 0 to 100 , and a total score of 0 20 suggested complete dependence, 21-60 severe dependence, 61-95 moderate dependence and 100 complete independence (18). The ADL questionnaire was answered by centenarians with health-conscious and checked by their relatives, and if they were incomplete health-conscious, the questionnaire was answered by their relatives (16). We defined ADL moderate and severe disability as reporting Barthel index score $\leq 60$, and ADL severe disability as reporting Barthel index score $\leq 40$ (19). The 8 items of Lawton IADL scale were summed to give a score from 0 to 8 , and participants scored 8 were defined as IADL independent, 6-7 were defined as IADL mild disability, $3-5$ were IADL moderate disability, and $\leq 2$ were IADL severe disability (20).

\section{Measurement of TC and Other Covariates}

The fasting blood was collected by experienced nurses using four vacutainer tubes $(2 \mathrm{ml})$ to detect the total cholesterol level. Blood specimens were placed in a cold box and then were tested in the Laboratory of Hainan Branch of the Chinese PLA General Hospital within $6 \mathrm{~h}$ by automatic biochemical analyzer. To better assess the association of TC with ADL disability, TC level was analyzed both as continuous and categorical variables in quartiles, respectively. TC level was divided into quartile: Q1 ( $\leq 4.05 \mathrm{mmol} / \mathrm{L}), \mathrm{Q} 2(4.06-4.60 \mathrm{mmol} / \mathrm{L}), \mathrm{Q} 3$ (4.61-5.25), and $\mathrm{Q} 4(\geq 5.26 \mathrm{mmol} / \mathrm{L})$.

Information on age, gender, nationality, marital status, educational level, and residential type were obtained through the questionnaire. Centenarians' disease history and whether taking 
TABLE 1 | Characteristics of total cholesterol level of hainan centenarians in China.

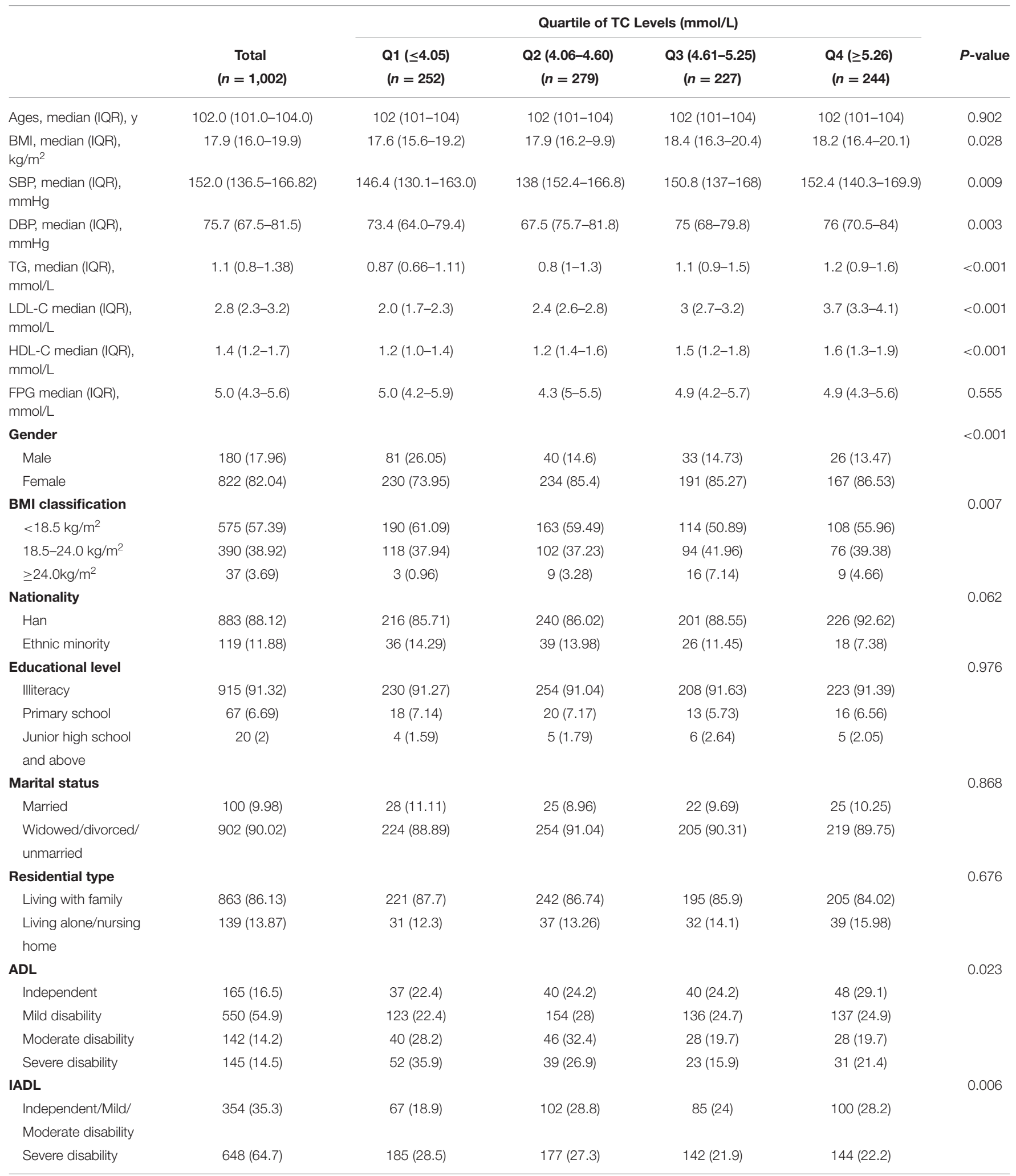

$A D L$, activities of daily living; IQR, interquartile range; BMI, body mass index; SBP, Systolic blood pressure; DBP, diastolic blood pressure; TG, triglyceride; FPG, fasting plasma glucose, $L D L-C$, low-density lipoprotein cholesterol; HDL-C, high-density lipoprotein cholesterol. 
medicine were surveyed. As most centenarians with develop a hunchback, the height of the centenarians was measured as the length from the top of the head through the spine to the heel with a standard soft ruler. Body Mass Index(BMI) categorized as underweight $\left(<18.5 \mathrm{~kg} / \mathrm{m}^{2}\right)$, normal weight $\left(18.5-24.0 \mathrm{~kg} / \mathrm{m}^{2}\right)$, and overweight $\left(\geq 24.0 \mathrm{~kg} / \mathrm{m}^{2}\right)(21)$. The blood pressure was measured twice in calm conditions by a portable electronic sphygmomanometer $(1 \mathrm{mmHg}=0.133 \mathrm{kPa})$ at intervals of 1-2 min. Smoking status, alcohol use, and physical activity were investigated by self-reported. The physical activity was assessed by answering "how many times did you do physical activities related to independent life per week," and we defined
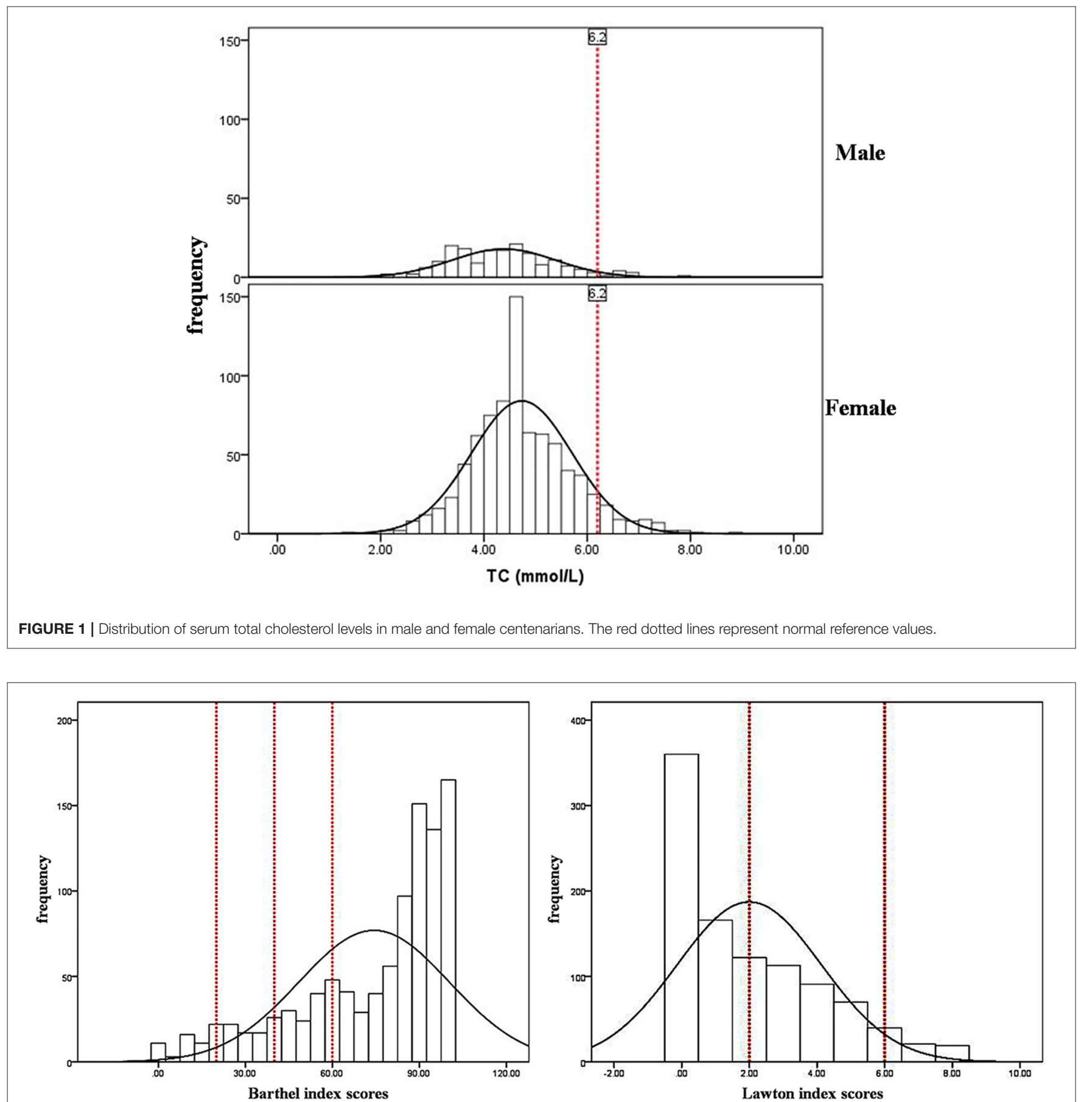

FIGURE 2 | Distribution of Barthel index scores and Lawton index scores in Hainan centenarians. The red dotted lines represent the cut-off value of different degrees of disability. 
infrequent activity as once weekly or less (22). The Mini-mental State Examination (MMSE) scale was used to estimate the cognition status.

\section{Statistical Analyses}

In this study, the Shapiro-Wilk test was used to estimate the normality of continuous variables and the results showed all continuous variables did not follow a normal distribution. Kruskal-Wallis test was used to test group differences, and the results were expressed as the median and interquartile range (IQR). The Chi-square test was used for intergroup comparison, and the results were expressed as number and percentage $(n / \%)$ since Barthel and Lawton scores following the non-normal distribution were analyzed as the categorical variables. The univariate and multivariable logistic regression models were used to explore the correlation between disability and TC levels. In multivariable analyses, demographic characteristics, health status, and physical activity were adjusted. We further excluded participants with dyslipidemia or taking lipid-lowering medicine to examine the possible correlation between disability and TC levels in sensitivity analyses. Odds Ratios (ORs) and 95\% confidence interval (CI) were reported. SPSS24.0 was used for statistical analysis and EmpowerStats (http://www.empowerstats. com, X\&Y Solutions, Inc., Boston, MA) and packages R (http:// www.R-project.org, The $\mathrm{R}$ Foundation) for restrictive cubic spline function. All $P$-values were from two-sided tests, and $P<$ 0.05 was used as the test level with statistical significance.

The possible mediations were explored according to the analytic methods outlined by Preacher and Hayes (23). All mediation analyses were carried out using PROCESS, which is an SPSS macro by Hayes $(24,25)$. The simple mediating effect was screened by PROCESS model 4 , and the multiple chain mediated model by PROCESS model 6 (25). All mediating effects were based on 5,000 samples bootstrapping set and all estimated mediating effects reported in this study are unstandardized regression coefficients.

TABLE 2 | The odds ratio of TC levels for disability in the multivariate logistic regression model.

ADL severe disability

\section{ALL CENTENARIANS $(n=1,002)$}

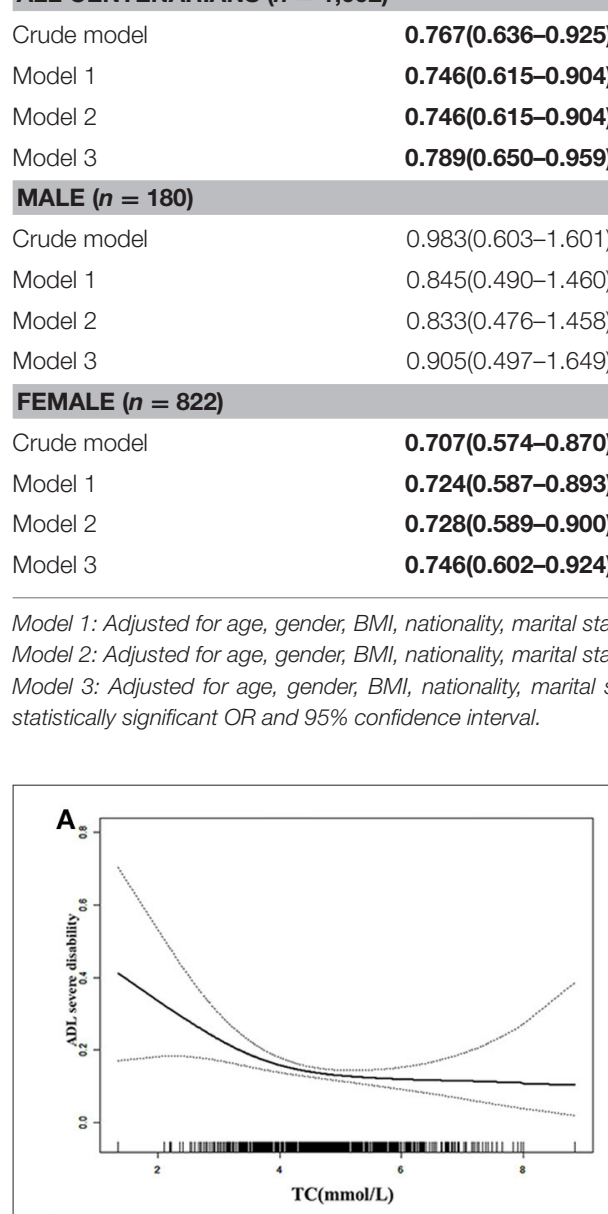
statistically significant OR and $95 \%$ confidence interval.

\section{$0.767(0.636-0.925)$

.

ADL moderate and severe disability

IADL severe disability

\section{ADL moderate and severe disability}

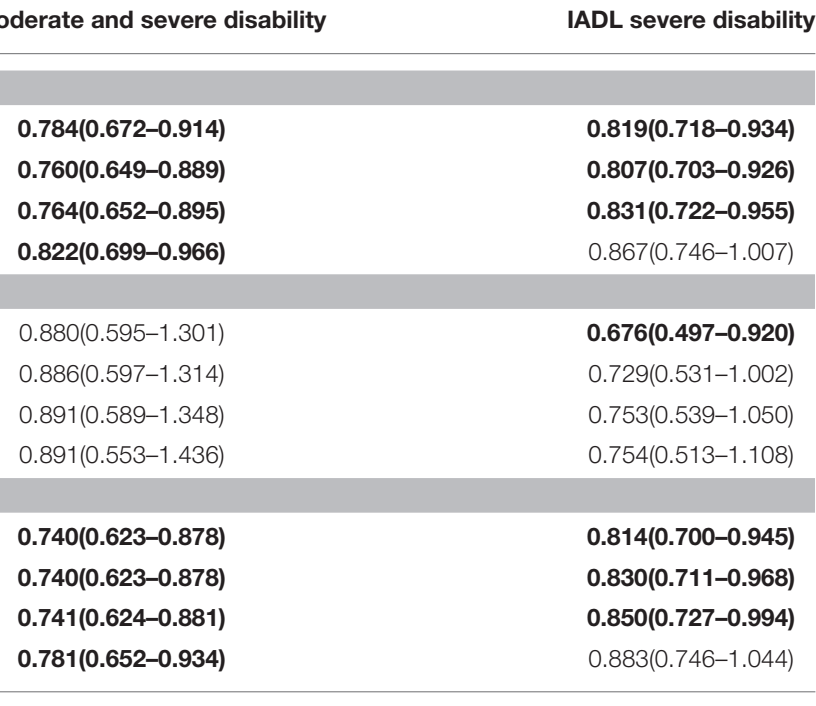

tus, educational level, and residential type.

Model 2: Adjusted for age, gender, BMI, nationality, marital status, educational level, residential type, glucose, and DBP.

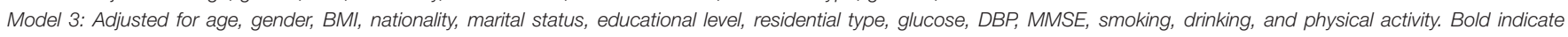
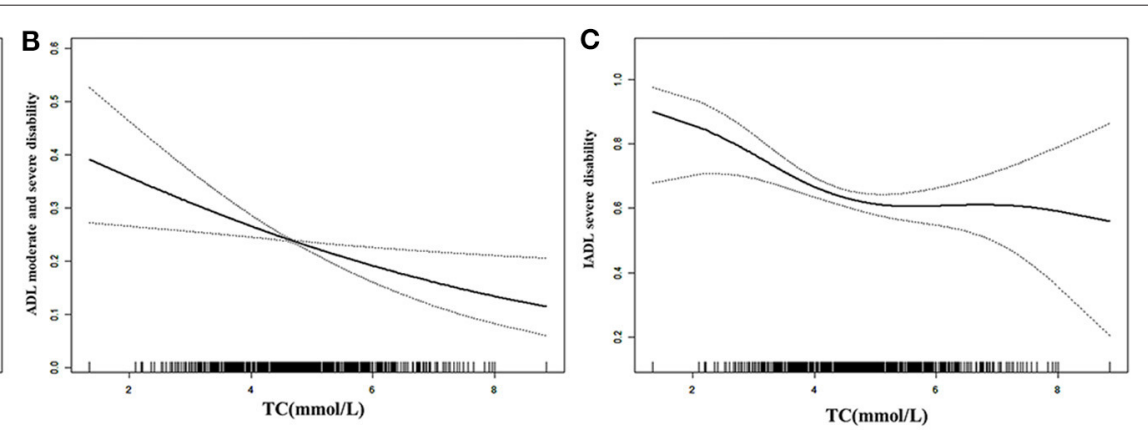

FIGURE 3 | Cubic spline curve of the inverse correlation between Total Cholesterol Levels and different types of disability [(A), ADL moderate and severe disability; (B), ADL severe disability; (C), IADL severe disability] in Hainan centenarians after adjusting for covariates. 


\section{RESULTS}

\section{Basic Characteristics of Centenarians}

Among 1,002 centenarians, the median age was 102 years (interquartile range [IQR], 101-104 years), and 822 were women (82.0\%). A total of 145 centenarians (14.5\%) were identified as ADL severe disability and 648 centenarians $(64.7 \%)$ as IADL severe disability (Table 1).

\section{Distribution of TC Level and ADL Scale}

Histogram showed the sex differences in the distribution of serum TC levels were significant and female centenarians had a higher level of TC (Figure 1). The TC median value in female centenarians $(4.60 \mathrm{mmol} / \mathrm{L})$ was higher than the median value in males $(4.35 \mathrm{mmol} / \mathrm{L})$. Histograms showed the distributions of Barthel index and Lawton index were significantly skewed distribution (Figure 2).

\section{The Correlations Between TC Levels and Disability}

The results of the multivariate Logistic regression model showed that, in the female centenarian population, after adjusting fully for potential confounding factors, the risk of ADL severe

TABLE 3 | The odds ratio (ORs) of TC categorical variables for ADL severe disability and ADL moderate and severe disability in the multivariate logistic regression model, compared the Q1.

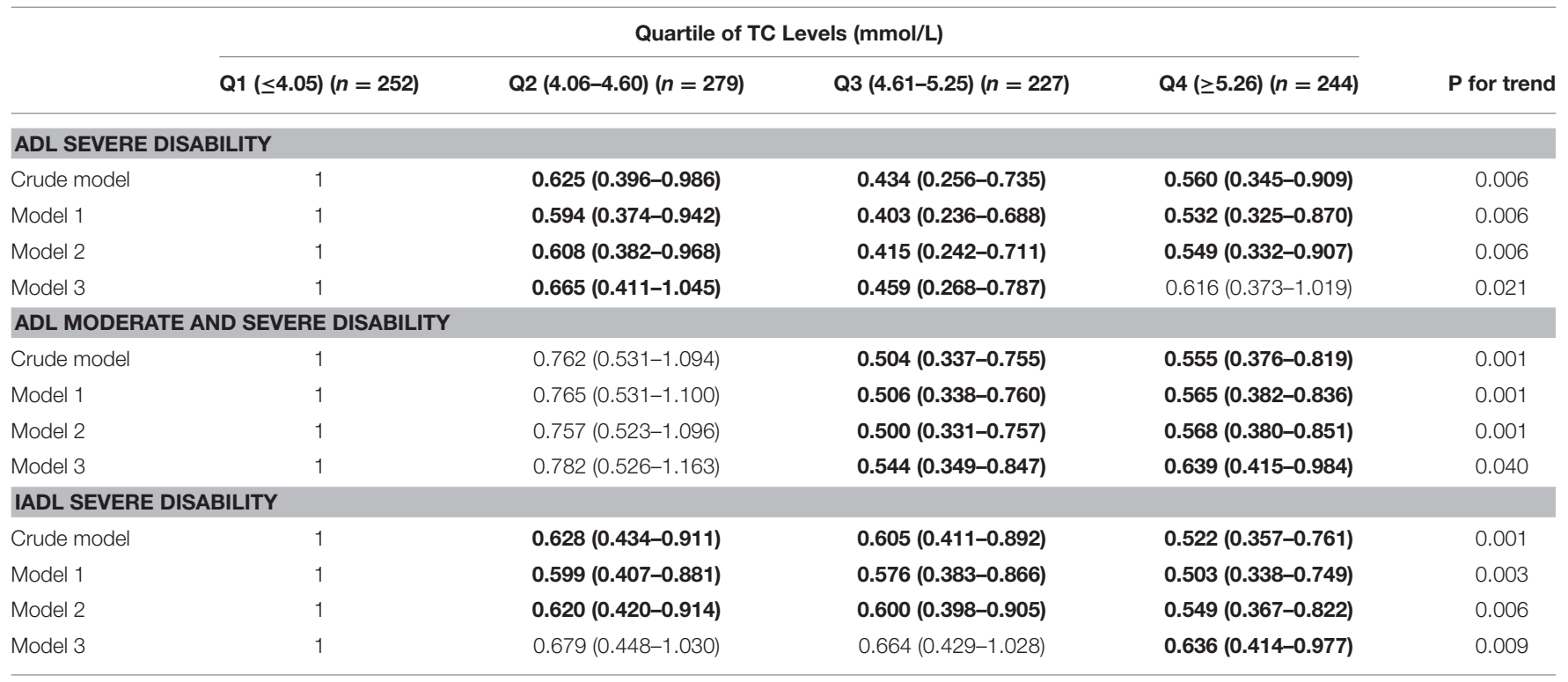

Model 1: Adjusted for age, BMI, nationality, marital status, educational level, and residential type.

Model 2: Adjusted for age, BMI, nationality, marital status, educational level, residential type, glucose, and DBP.

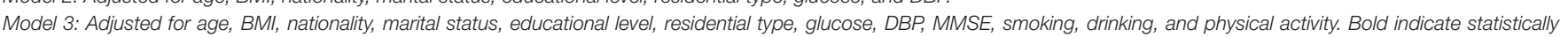
significant $O R$ and $95 \%$ confidence interval.

TABLE 4 | Correlations of TC indicators and disability among female Hainan 435 centenarians without dyslipidemia, previous hyperlipidemia, and taking lipid-lowering medicine $^{\mathrm{a}}$.

TC Level

\begin{tabular}{|c|c|c|c|c|c|c|}
\hline & TC Level & \multicolumn{5}{|c|}{ TC Quartile of Female Centenarians ( $n=736$ ) } \\
\hline Unadjusted & $0.654(0.507-0.842)$ & 1 & $0.334(0.183-0.612)$ & $0.593(0.350-1.004)$ & $0.526(0.306-0.905)$ & 0.001 \\
\hline Adjusted $^{\mathrm{b}}$ & & 1 & $0.345(0.183-0.652)$ & $0.600(0.341-1.056)$ & $0.554(0.308-0.996)$ & 0.107 \\
\hline \multicolumn{7}{|c|}{ ADL MODERATE/SEVERE DISABILITY } \\
\hline \multicolumn{7}{|c|}{ IADL SEVERE DISABILITY } \\
\hline Unadjusted & $0.787(0.644-0.963)$ & 1 & $0.580(0.369-0.910)$ & $0.603(0.383-0.948)$ & $0.639(0.404-1.006)$ & 0.020 \\
\hline Adjusted $^{\mathrm{b}}$ & & 1 & $0.642(0.375-1.101)$ & $0.609(0.358-1.038)$ & $0.751(0.434-1.299)$ & 0.317 \\
\hline
\end{tabular}

${ }^{a}$ Results weighted to sensitivity analysis estimates which have been balanced the complex confounding factors.

${ }^{b}$ All models adjusted for age, BMI, nationality, marital status, educational level, residential type, glucose, DBP, MMSE, smoking, drinking, and physical activity. 
disability, ADL moderate and severe disability decreased by $21.1 \%(\mathrm{OR}=0.789,95 \% \mathrm{CI}: 0.650-0.959)$ and $27.8 \%(\mathrm{OR}=0.822$, 95\%CI:0.699-0.966), respectively, with the increment of $1 \mathrm{mmol}$ / L of TC level. No significant correlation was found in male centenarians (Table 2 and Figure 3 ).

\section{The Correlations Between TC Quartile and Disability}

After adjustment, compared to centenarians with TC 1st quartile, the risk of TC 4th quartile of ADL moderate and severe disability and IADL severe disability decreased by $46.1 \%(\mathrm{OR}=0.639$, 95\%CI: $0.415-0.984$ ), and 36.4\% (OR = 0. 636, 95\%CI: $0.414-$ 0.977), respectively (Table 3 ).

\section{Sensitivity Analysis}

We also analyzed the association of TC levels with disability among 710 female centenarians without dyslipidemia who were taking lipid-lowering medicine. After adjustment, the risk of ADL severe disability, ADL moderate and severe disability, and IADL severe disability decreased with the increment of TC indicators (Table 4).

\section{The Mediation Analysis}

We examined, among Chinese female centenarians, whether BMI and blood pressure indicators (SBP and DBP) mediated the relationship between TC levels and different types of disability (ADL moderate/severe disability, ADL severe disability, and IADL severe disability), respectively, adjusting for the age, nationality, educational level, marital status, residential type, FPG, MMSE, smoking, and drinking. The results of mediating effect indicated lower serum lipid, lower blood pressure, and lower BMI may be correlated with functional impairment in female centenarians, and simple mediating effect showed BMI mediating the effect of TC levels on different types of disability (ADL moderate/severe disability, ADL severe disability and IADL severe disability) was significant, accounted for
$6.17,6.10$, and $7.42 \%$ of the total effect, respectively (Table 5 and Figure 4).

After adjusting for covariates, the results of the multiple chain mediated model showed a significant chain mediating effect through the path completely accomplished by chain mediators BMI and SBP $(B=0.0013, P=0.0010)$, and two significant simple mediating paths mediated by $\mathrm{BMI}(\mathrm{B}=0.0158, P=$ $0.0131)$, and SBP $(B=0.0139, P=0.0085)$, respectively. The effect of TC on IADL was completely mediated by BMI and SBP (Table 6 and Figure 5).

\section{DISCUSSION}

The main finding from this cross-sectional and complete samples study on Hainan centenarians suggest that elevated TC was inversely correlated with disability, and the association might be mediated by BMI and SBP in the female centenarian population. To our knowledge, this is the first study describing the epidemiological correlation between TC level and disability, and to quantify the mediating effect of this relationship among a complete sample female centenarian population.

Similar to other studies, our study found that, in Hainan centenarians, the prevalence of disability was high and the TC levels were moderate $(26,27)$. The variability among different studies might be attributed to different ADL scales. Several studies have confirmed that the TC level in centenarians was generally within the normal range, and was lower than that in the healthy older adults control group $(28,29)$.

In this study, the elevated TC levels in female Hainan centenarians were correlated with disability among centenarians. This possible relationship was still stable in sensitivity analyses. However, the association we deduced was inconsistent with those concluded among the different age groups. A 32-year follow-up survey from Chicago Heart Association Detection Project suggested that lower TC levels were associated with the

TABLE 5 | The mediating effect of BMl between TC levels and different types of disability.

\begin{tabular}{|c|c|c|c|c|c|c|}
\hline & Coefficient & Standard deviation & t-value & $P$ & Total effect & Mediating effect \\
\hline ADL moderate/severe disability & & & & & -0.2594 & -0.0160 \\
\hline $\mathrm{TC} \rightarrow$ disability & -0.2594 & 0.0981 & -2.7488 & 0.0044 & & \\
\hline $\mathrm{TC} \rightarrow \mathrm{BMl}$ & 0.2853 & 0.1159 & 2.4615 & 0.0140 & & \\
\hline $\mathrm{BMl} \rightarrow$ disability & -0.0561 & 0.0270 & -2.0792 & 0.0376 & & \\
\hline TC' $\rightarrow$ disability & -0.2434 & 0.0897 & -2.7131 & 0.0067 & & \\
\hline ADL severe disability & & & & & -0.3082 & -0.0188 \\
\hline $\mathrm{TC} \rightarrow$ disability & -0.3082 & 0.1200 & -2.5198 & 0.0050 & & \\
\hline $\mathrm{TC} \rightarrow \mathrm{BMl}$ & 0.2853 & 0.1159 & 2.4615 & 0.0140 & & \\
\hline $\mathrm{BMl} \rightarrow$ disability & -0.0658 & 0.0324 & -2.0297 & 0.0424 & & \\
\hline TC' $\rightarrow$ disability & -0.2894 & 0.1076 & -2.6901 & 0.0071 & & \\
\hline IADL severe disability & & & & & -0.2063 & -0.0153 \\
\hline $\mathrm{TC} \rightarrow$ disability & -0.2063 & 0.0764 & -2.6995 & 0.0071 & & \\
\hline $\mathrm{TC} \rightarrow \mathrm{BMl}$ & 0.2853 & 0.1159 & 2.4615 & 0.0140 & & \\
\hline $\mathrm{BMI} \rightarrow$ disability & -0.0536 & 0.0245 & -2.1872 & 0.0287 & & \\
\hline TC' $\rightarrow$ disability & -0.1485 & 0.0828 & -1.7928 & 0.0730 & & \\
\hline
\end{tabular}



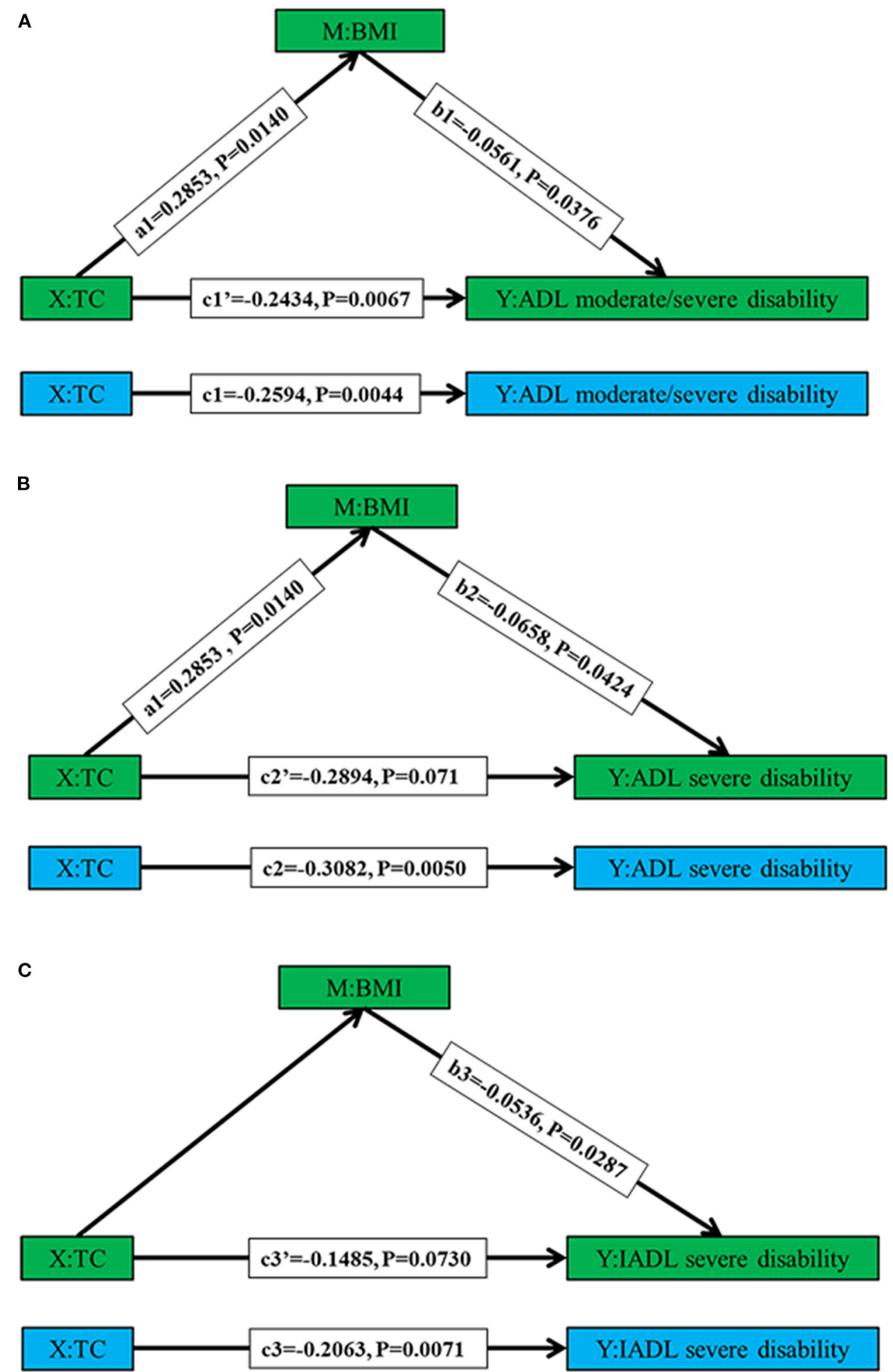

FIGURE 4 | The simple mediating effects of BMI between TC levels $(X)$ and different types of disability $(Y)$. The blue graphs depicting the total effect (c) of TC on different types of disability $(\mathrm{Y})$, and the green graphs depicting the direct effect ( $\mathrm{C}^{\prime}$ ) and mediating effects of BMI on TC on different types of disability ( $\mathrm{Y}$ ). Graph $\mathbf{A}-\mathbf{C}$ representing different types of disability (ADL moderate/severe disability, ADL severe disability, and IADL severe disability), respectively. Effect values refer to unstandardized regression coefficients. 
lowest rate of functional disability in older age (30). Among the younger- and older-old from Sweden, the results suggested there was no statistical correlation between blood lipids and ADL disability (31). Lower total cholesterol and high-density lipoprotein cholesterol levels were associated with a higher risk of $\mathrm{ADL}$ disability $(32,33)$.

Inconsistent correlations might be partially attributable to age difference in that the old with higher serum lipid levels were more likely to die early from cardiovascular diseases, and it might be also explained that centenarians might have unique physiological reference values of blood lipids profile, which was significantly different from that of younger adults, but the current physiological reference standards for blood lipid profile was not standardized according to age (34). The inverse correlation between elevated TC level and disability was only found in female centenarians. Gender differences may be mainly caused by different physiological characteristics (34).

TABLE 6 | The effect values of mediating paths from TC to IADL in the multiple chain mediated model among female centenarians.

\begin{tabular}{lccc}
\hline Paths & Coefficient & $\mathbf{9 5 \%} \mathbf{C l}$ & $\boldsymbol{P}$ \\
\hline $\mathrm{TC} \rightarrow \mathrm{BMI}$ & 0.2853 & $0.0578-0.5128$ & 0.0140 \\
$\mathrm{TC} \rightarrow \mathrm{SBP}$ & 2.1234 & $0.5354-3.7114$ & 0.0088 \\
$\mathrm{BMI} \rightarrow \mid \mathrm{ADL}$ scores & 0.0553 & $0.0164-0.0941$ & 0.0054 \\
$\mathrm{SBP} \rightarrow \mathrm{IADL}$ scores & 0.0066 & $0.0010-0.0121$ & 0.0209 \\
$\mathrm{BMl} \rightarrow \mathrm{SBP}$ & 0.7041 & $0.2271-1.1811$ & 0.0039 \\
$\mathrm{TC} \rightarrow \mathrm{ADL}$ scores & 0.0967 & $-0.0326-0.2259$ & 0.1425 \\
$\mathrm{TC} \rightarrow \mathrm{BMI} \rightarrow \mid \mathrm{ADL}$ scores & 0.0158 & $0.0022-0.0357$ & 0.0131 \\
$\mathrm{TC} \rightarrow \mathrm{BMI} \rightarrow \mathrm{SBP} \rightarrow \mid \mathrm{ADL}$ scores & 0.0013 & $0.0001-0.0037$ & 0.0010 \\
$\mathrm{TC} \rightarrow \mathrm{SBP} \rightarrow \mid \mathrm{ADL}$ scores & 0.0139 & $0.0010-0.0329$ & 0.0085
\end{tabular}

The effect value of mediating paths of $T C \rightarrow B M I \rightarrow I A D L$ scores is the product of $T C \rightarrow B M I$ and $B M I \rightarrow I A D L$ scores; The effect value of mediating paths of $T C \rightarrow S B P \rightarrow I A D L$ scores is the product of $T C \rightarrow S B P$ and $S B P \rightarrow I A D L$ scores; The effect value of mediating paths of $T C \rightarrow B M I \rightarrow S B P \rightarrow I A D L$ scores is the product of $T C \rightarrow B M I, B M I \rightarrow S B P$ and $B M I \rightarrow I A D L$ scores.
The previous studies have shown that older adults' functional impairment might be related to malnutrition $(35,36)$. In the current study, TC and BMI levels can represent some nutritional status of the centenarians. Lipids profile is influenced by comprehensive, whole-life cycle heredity, diet, and nutrition. BMI can reflect the degree of nutrition and obesity. 57.39\% of Hainan centenarians were underweight, and $7 \%$ were hypercholesterolemia. Centenarians have a thinner body shape and lower TC levels than those adults. BMI was positively correlated to serum the TC level. It was suggested that the possible correlation between the BMI and TC levels may be significant, both as indicators for assessing the nutrition status. Therefore, in this study, BMI was considered as a confounding factor to be adjusted in the multivariate logistic regression model and treated as a possible mediator in mediating effect analysis.

The results of the simple mediating effect suggested that BMI mediated the influence of TC on disabilities, which meant the higher the TC level, the higher the BMI, and the latter was related to less likely the risk of disability. The effects of TC on ADL disabilities were partially mediated by BMI. The results of the multiple-chain-mediating effect suggested BMI and SBP completely mediated this association either singly or in combination, which indicated the level of total cholesterol might indirectly and variously affect the disability through BMI and SBP. At present, no similar studies have been found and the causality deserves further verification. Nevertheless, some interesting perspectives for future research were presented. The potential public health meanings were that serum lipid, BMI, and blood pressure should be further noticed among the extreme longevity.

Although the exact mechanism remains unclear, several plausible mechanisms may underlie the inverse correlation between TC levels and ADL disability. First, cholesterol is involved in many important biochemical pathways (37), which may indirectly affect the ADLs of centenarians (38). Secondly, inflammatory markers associated with low total cholesterol levels were found to be associated with disability and poor Barthel ADL scores in older people (39). Cholesterol and its metabolites may

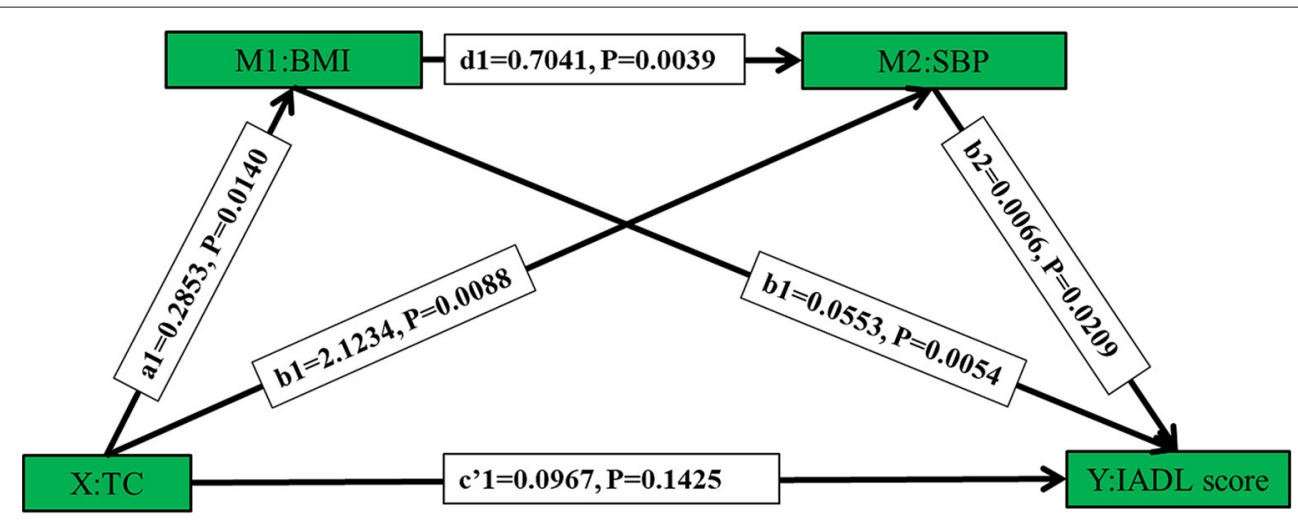

FIGURE 5 | The multiple-chain-mediated model, including multiple mediating effect and chain mediating effects, depicting direct and indirect effects of TC levels ( $X$ ) on IADL scores (Y). BMI and SBP, as a mediator as well as chain mediators, partially mediate the effect of total cholesterol on disability. Effect values refer to unstandardized regression coefficients. 
regulate interleukin-6 signaling (40). Thirdly, cholesterol plays an important role in the formation and deposition of synapses, membranes, and amyloid- $\beta$, which affect brain function (41).

Our findings, similar to other studies, found the gender differences between TC and disability in centenarians. The results from the Chinese Longitudinal Healthy Longevity Survey (CLHLS) indicated that higher levels of total cholesterol and triglyceride within the normal range had protective effects on functional impairment of older people and particularly females $(42,43)$. We postulated that inversely associations of TC levels and ADL disability among female centenarians are as follows: (1) it is believed that women live longer in extremely long-lived populations, but men exhibit higher levels of cognitive function and ADL (44-46); (2) survivor bias, women were more likely to experience the higher prevalence of disability, was hard to rule out.

This study has some limitations. The conclusions that emerged from this cross-sectional study are not the real causal links between TC level and disability among centenarians but raise several intriguing speculations in such an extreme longevity population for further identification and research. Second, along with the Chinese nationality, there are possible associations of this study that are less generalizable to other regions and populations. Third, the results of the Barthel index and Lawton scale were self-reported. Bias would be difficult to avoid and was likely to occur. Fourth, due to the lower prevalence of hypercholesterolemia in centenarians, TC level was quartered to better describe its distribution characteristics, which was biased that the results of this quartering method only represented this centenarian population and could not explain the health impact of hypercholesterolemia on ADLs.

\section{CONCLUSION}

This study is the first to demonstrate that low TC levels might be correlated with a higher frequency of disability in female centenarians, and this correlation might be mediated by BMI and blood pressure either singly or in combination. Accordingly, we hypothesize that lower serum lipid, blood pressure, and BMI may be detrimental to function in centenarians. Significantly, our positive results emerged from a cross-sectional design that could not draw any causal conclusions, and even the inversion of cause and effect cannot be ruled out. But these possible links, between

\section{REFERENCES}

1. Fuster V. Changing demographics: a new approach to global health care due to the aging population. J Am Coll Cardiol. (2017) 69:3002. doi: 10.1016/j.jacc.2017.05.013

2. Li J, Xu X, Sun J, Cai W, Qin T, Wu M, et al. Activities of daily living, life orientation, and health-related quality of life among older people in nursing homes: a national cross-sectional study in China. Qual Life Res. (2020) 29:2949-60. doi: 10.1007/s11136-020-02571-w

3. Fries JF. Aging, natural death, and the compression of morbidity. (1980) 303:1369-70. doi: 10.1056/NEJM198012043032317

4. Zeng Y, Feng Q, Hesketh T, Christensen K, Vaupel JW. Survival, disabilities in activities of daily living, and physical and cognitive functioning among disability and its related serum lipid indicators, may provide a way of thinking and hypothesis to be explored and identified.

\section{DATA AVAILABILITY STATEMENT}

The datasets China Hainan Centenarians Cohort Study (CHCCS) for this study was available upon request.

\section{ETHICS STATEMENT}

The studies involving human participants were reviewed and approved by the Ethics Committee of Chinese People's Liberation Army General Hospital (approval number: 301hn11201601). The patients/participants provided their written informed consent to participate in this study.

\section{AUTHOR CONTRIBUTIONS}

SW, WJ, and SY were involved in the conception and design of the work. SW and WJ contributed to writing the manuscript. $\mathrm{KH}, \mathrm{WC}$, and $\mathrm{XR}$ contributed to data arrangement and statistical analysis. SY, WC, and WJ contributed to the data compilation. $\mathrm{ML}, \mathrm{XR}$, and YH contributed to critical review. PT, FK, and JL contributed to the literature search. SY, WJ, and SW contributed to the design of tables and figures. All authors contributed to the article and approved the submitted version.

\section{FUNDING}

This study was funded by Beijing Nova Program (Z181100006218085), Opening Foundation of State Key Laboratory of Kidney Diseases (KF-01-115), Opening Foundation of National Clinical Research Center of Geriatrics (NCRCG-PLAGH-2017017), National Natural Science Foundation of China (81773502, 81703285, and 81703308), Medical Big Data Fund of Chinese PLA General Hospital (2018MBD-029).

\section{ACKNOWLEDGMENTS}

The authors thank all the centenarians and staff involved in the China Hainan Centenarians Cohort Study (CHCCS). the oldest-old in China: a cohort study. Lancet. (2017) 389:1619-29. doi: 10.1016/S0140-6736(17)30548-2

5. Mahoney FI, Barthel DW. Functional evaluation: the barthel index. Md State Med J. (1965) 14:61-5. doi: 10.1037/t02366-000

6. Lawton MP, Brody EM. Assessment of older people: self-maintaining and instrumental activities of daily living. The Gerontologist. (1969) 9:179-86. doi: 10.1093/geront/9.3_Part_1.179

7. Daviglus ML, Talavera GA, Avilés-Santa ML, Matthew A, Jianwen C, Criqui $\mathrm{MH}$, et al. Prevalence of major cardiovascular risk factors and cardiovascular diseases among Hispanic/Latino individuals of diverse backgrounds in the United States. JAMA. (2012) 308:1775-84. doi: 10.1001/jama.2012.14517

8. Quanhe Y, Cogswell ME, Dana F, Yuling H, Zefeng Z, Fleetwood L, et al. Trends in cardiovascular health metrics and associations with 
all-cause and CVD mortality among US adults. JAMA. (2012) 307:1273. doi: 10.1001/jama.2012.339

9. Reed B, Villeneuve S, Mack W, Decarli C, Chui HC, Jagust WJJN. Associations between serum cholesterol levels and cerebral amyloidosis. JAMA Neurol. (2014) 71:195-200. doi: 10.1001/jamaneurol.2013.5390

10. Carriere I, Pérès $\mathrm{K}$, Ancelin $\mathrm{M} \mathrm{L}$, Gourlet V, Berr C, BarbergerGateau P, et al. Metabolic syndrome and disability: findings from the prospective three-city study. J Gerontol A Biol Sci Med Sci. (2014) 69:79-86. doi: 10.1093 /gerona/glt101

11. Lv YB, Mao C, Gao X, Yin ZX, Kraus VB, Yuan JQ, et al. Triglycerides paradox among the oldest old: "the lower the better?". J Am Geriatr Soc. (2019) 67:741-8. doi: 10.1111/jgs.15733

12. Wang S, Liu M, Yang S, Wang J, Jia W, Cao W, et al. Higher normal levels of triglyceride and low and high-density lipoprotein cholesterol might have a protective effect against activities of daily living disability within chinese female centenarians: a cross-sectional, complete sample study. Clin Interv Aging. (2020) 15:225-37. doi: 10.2147/cia.S237505

13. Ravnskov U, Diamond DM, Hama R, Hamazaki T, Hammarskjöld B, Hynes $\mathrm{N}$, et al. Lack of an association or an inverse association between low-densitylipoprotein cholesterol and mortality in the elderly: a systematic review. $B M J$ Open. (2016) 6:e010401. doi: 10.1136/bmjopen-2015-010401

14. Chanti-Ketterl M, Gamaldo A, Andel RThorpe RJ Jr. The association between lipoproteins, disability, and physical function among older costa rican adults. J Aging Health. (2018) 30:758-77. doi: 10.1177/08982643176 90866

15. Choi H, Kim HC, Stefani KM, Lee JM, Yoon YM, Lee EY, et al. Serum highdensity lipoprotein cholesterol concentration and functional state: the Korean Urban Rural Elderly (KURE) Study. Arch Gerontol Geriatr. (2017) 71:115-21. doi: 10.1016/j.archger.2017.04.002

16. He Y, Zhao Y, Yao Y, Yang S, Li J, Liu M, et al. Cohort profile: the china hainan centenarian cohort study (CHCCS). Int J Epidemiol. (2018) 47:694-5. doi: 10.1093/ije/dyy017

17. Brown RT, Diaz-Ramirez LG, Boscardin WJ, Lee SJ, Williams BA, Steinman MA. Association of functional impairment in middle age with hospitalization, nursing home admission, and death. JAMA Intern Med. (2019) 179:668-75. doi: 10.1001/jamainternmed.2019.0008

18. Dromerick AW, Edwards DF, Diringer MNJJoRR. Development. Sensitivity to changes in disability after stroke: a comparison of four scales useful in clinical trials. J Rehabil Res Dev. (2003) 40:1. doi: 10.1682/JRRD.2003. 01.0001

19. Wade DT, Collin C. The Barthel ADL index: a standard measure of physical disability? International Rehabilitation Medicine. Int Disabil Stud. (1988) 10:64-7. doi: 10.3109/09638288809164105

20. Li JQ, Liu M, Lyu XY, Tang R, Yang X, Yin $M$, et al. [Prevalence of chronic diseases and associate factors on daily activities in male oldest-olds]. Zhonghua Liu Xing Bing Xue Za Zhi. (2019) 40:537-41. doi: 10.3760/cma.j.issn.0254-6450.2019.05.009

21. Department of Disease Control, Ministry of Health of the People's Republic of China. The Guideline of the Prevention and Control of Overweight and Obesity in Chinese Adults [M]. Department of Disease Control, Ministry of Health of the People's Republic of China: People's Medical Publishing House (2006).

22. He XZ, Baker DW. Body mass index, physical activity, and the risk of decline in overall health and physical functioning in late middle age. Am J Public Health. (2004) 94:1567. doi: 10.2105/AJPH.94.9.1567

23. Preacher KJ, Hayes AF. Asymptotic and resampling strategies for assessing and comparing indirect effects in multiple mediator models. Behav Res Methods. (2008) 40:879-91. doi: 10.3758/brm.40.3.879

24. Hayes AF, Rockwood NJ. Regression-based statistical mediation and moderation analysis in clinical research: Observations, recommendations, and implementation. Behav Res Ther. (2017) 98:39-57. doi: 10.1016/j.brat.2016.11.001

25. Preacher KJ, Hayes AF. SPSS and SAS procedures for estimating indirect effects in simple mediation models. Behav Res Methods Instrum Comput. (2004) 36:717-31. doi: 10.3758/bf03206553

26. Berlau DJ, Corrada MM, Peltz CB, Kawas CH. Disability in the oldest-old: incidence and risk factors in the 90+ study. Am J Geriatr Psychiatry. (2012) 20:159-68. doi: 10.1097/JGP.0b013e31820 d 9295
27. Wu H, Flaherty J, Dong B, Liu G, Deng J, Zhang Y, et al. Impact of geriatric conditions versus medical diagnoses on ADL disability among nonagenarians and centenarians. J Aging and Health. (2012) 24:1298-319. doi: $10.1177 / 0898264312457411$

28. Lio D, Malaguarnera M, Maugeri D, Ferlito L, Bennati E, Scola L, et al. Laboratory parameters in centenarians of Italian ancestry. Experimental Gerontol. (2008) 43:119-22. doi: 10.1016/j.exger.2007.06.005

29. Baranowska B, Bik W, Baranowska-Bik A, Wolinska-Witort E, Szybinska A, Martynska L, et al. Neuroendocrine control of metabolic homeostasis in Polish centenarians. J Physiol Pharmacol. (2006) 57(Suppl 6):55-61. Available online at: https://europepmc.org/article/med/17228087

30. Vu TH, Lloyd-Jones DM, Liu K, Stamler J, Garside DB, Daviglus ML. Optimal levels of all major cardiovascular risk factors in younger age and functional disability in older age: the chicago heart association detection project in industry 32-year follow-up health survey. Circ Cardiovasc Qual Outcomes. (2016) 9:355-63. doi: 10.1161/circoutcomes.115.002519

31. Heiland EG, Welmer AK, Wang R, Santoni G, Fratiglioni L, Qiu C. Cardiovascular risk factors and the risk of disability in older adults: variation by age and functional status. J Am Med Dir Assoc. (2019) 20:208-12.e3. doi: 10.1016/j.jamda.2018.05.013

32. Tomonori O, Takehito H, Atsushi H, Takashi K, Yoshitaka M, Yoshikuni $\mathrm{K}$, et al. Lower levels of serum albumin and total cholesterol associated with decline in activities of daily living and excess mortality in a 12year cohort study of elderly Japanese. J Am Geriatr Soc. (2010) 56:529-35. doi: 10.1111/j.1532-5415.2007.01549.x

33. Zuliani G, Romagnoni F, Bollini C, Leoci V, Soattin L, Fellin R. Low levels of high-density lipoprotein cholesterol are a marker of disability in the elderly. Gerontology. (1999) 45:317-22. doi: 10.1159/000022112

34. Barzilai N, Atzmon G, Schechter C, Schaefer EJ, Cupples AL, Lipton $\mathrm{R}$, et al. Unique lipoprotein phenotype and genotype associated with exceptional longevity. JAMA. (2003) 290:2030-40. doi: 10.1001/jama.290. 15.2030

35. Verlaan S, Ligthart-Melis GC, Wijers LJ, Cederholm T, Maier AB, de van der Schueren MAE. High prevalence of physical frailty among communitydwelling malnourished older adults-a systematic review and meta-analysis. J Am Med Dir Assoc. (2017) 18:374-382. doi: 10.1016/j.jamda.2016. 12.074

36. Vandewoude MFJ, van Wijngaarden JP, De Maesschalck L, Luiking YC, Van Gossum A. The prevalence and health burden of malnutrition in Belgian older people in the community or residing in nursing homes: results of the NutriAction II study. Aging Clin Exp Res. (2019) 31:17583. doi: 10.1007/s40520-018-0957-2

37. Jorde R, Grimnes G. Vitamin D and metabolic health with special reference to the effect of vitamin D on serum lipids. Prog Lipid Res. (2011) 50:303-12. doi: 10.1016/j.plipres.2011.05.001

38. Eliassen AH, Hankinson SE. Endogenous hormone levels and risk of breast, endometrial and ovarian cancers: prospective studies. Adv Exp Med Biol. (2008) 630:148-65. doi: 10.1007/978-0-387-78818$0 \_10$

39. Cesari M, Penninx BW, Pahor M, Lauretani F, Corsi AM, Rhys Williams $\mathrm{G}$, et al. Inflammatory markers and physical performance in older persons: the InCHIANTI study. J Gerontol A Biol Sci Med Sci. (2004) 59:242-8. doi: 10.1093/gerona/59.3.m242

40. Omoigui S. Cholesterol synthesis is the trigger and isoprenoid dependent interleukin-6 mediated inflammation is the common causative factor and therapeutic target for atherosclerotic vascular disease and age-related disorders including osteoporosis and type 2 diabetes. Med Hypotheses. (2005) 65:559-69. doi: 10.1016/j.mehy.2005.03.012

41. van Vliet P. Cholesterol and late-life cognitive decline. J Alzheimers Dis. (2012) 30(Suppl 2):S147-62. doi: 10.3233/jad-2011-111028

42. Lv YB, Yin ZX, Chei CL, Brasher MS, Zhang J, Kraus VB, et al. Serum cholesterol levels within the high normal range are associated with better cognitive performance among Chinese elderly. J Nutr Health Aging. (2016) 20:1-8. doi: 10.1007/s12603-016-0701-6

43. Zhao-Xue Y, Xiao-Ming S, Kraus VB, Fitzgerald SM, Han-Zhu Q, Jian-Wei $\mathrm{X}$, et al. High normal plasma triglycerides are associated with preserved cognitive function in Chinese oldest-old. Age Ageing. (2012) 41:600-6. doi: 10.1093/ageing/afs033 
44. Kim H, Lee T, Lee S, Kim K, Lee S, Kam S, et al. Factors associated with ADL and IADL dependency among Korean centenarians: reaching the 100year-old life transition. Int J Aging Hum Dev. (2012) 74:243. doi: 10.2190/ AG.74.3.e

45. Yang M, Hao Q, Luo L, Ding X, Wu H, Zhang Y, et al. Body mass index and disability in chinese nonagenarians and centenarians. J Am Med Dir Assoc. (2014) 15:303.e1-e6. doi: 10.1016/j.jamda.2013.10.011

46. Hao Z, Chen L, Li Y, Zou X, Li H, Feng Z, et al. Characteristics of centenarians' lifestyles and their contribution to life satisfaction: a case study conducted on Hainan Island. Arch Gerontol Geriatr. (2019) 83:20-7. doi: 10.1016/j.archger.2019.03.022
Conflict of Interest: The authors declare that the research was conducted in the absence of any commercial or financial relationships that could be construed as a potential conflict of interest.

Copyright (C) 2021 Wang, Jia, Yang, Han, Cao, Ren, Li, Tai, Kou, Liu and He. This is an open-access article distributed under the terms of the Creative Commons Attribution License (CC BY). The use, distribution or reproduction in other forums is permitted, provided the original author(s) and the copyright owner(s) are credited and that the original publication in this journal is cited, in accordance with accepted academic practice. No use, distribution or reproduction is permitted which does not comply with these terms. 